Methods 48 CYP-Treg cell lines were obtained from $12 \mathrm{AIH}-2$ patients positive for the predisposing HLA-DR7 and DR3 alleles; 36 Treg cell lines specific for a DR7 or DR3-restricted influenzahaemagglutinin (HA) peptide were generated from $9 \mathrm{DR}^{+}$or $\mathrm{DR}^{+}$ healthy subjects (HS) and used as controls. CYP- and HA-Treg were obtained after co-culture with peptide-pulsed semi-mature DCs. T-reg were expanded for 2 weeks in the presence of: (1) IL2 (300 U/ $\mathrm{ml}$ ); (2) IL2 + rapamycin (RP) to enhance Treg function; (3) IL2+IL6/ IL1b, cytokines mimicking the proinflammatory milieu of AIH-2. Treg phenotype was determined by flow cytometry; frequency of cytokine-producing cells by intracellular staining.

Results Before expansion, the frequency of $\mathrm{CD}_{127^{-}}$and $\mathrm{FOXP3}^{+}$ cells exceeded $80 \%$ in both CYP- and HA-Treg. Compared to HATreg, CYP-Treg contained higher numbers of IFNg (6.4 \pm 1 vs $3.6 \pm 1.2, \mathrm{p}=0.09)$, IL2 (9.5 \pm 2.6 vs $2.6 \pm 0.5, \mathrm{p}=0.02)$, IL17 (7.1 \pm 1 vs $3 \pm 1.2, \mathrm{p}=0.026)$, IL10 (9.1 \pm 2 vs $3.3 \pm 2.6, \mathrm{p}=0.03)$ and TGFb $(10.4 \pm 2$ vs 3.6 $\pm 0.7, p=0.001)$ producing cells. After expansion with IL2, CYP- and HA-Treg maintained a similarly high frequency of $\mathrm{FOXP3}^{+}$and $\mathrm{CD} 127^{-}$cells, while frequency of $\mathrm{IFNg}^{+}$cells increased markedly (CYP-Treg: from $6.5 \pm 1$ to $27 \pm 3, \mathrm{p}<0.0001$; HA-Treg: from $3.8 \pm 1.5$ to $11 \pm 1.3, p<0.01)$. Exposure to RP decreased the frequency of IFNg ${ }^{+}$cells by $36 \%(p=0.04)$ in HS and by $30 \%(p=0.15)$ in AIH2. Exposure of Treg to IL6/IL1b had no effect on their phenotype and cytokine production.

Conclusion Compared to HA-Treg, CYP-Treg contain higher numbers of cytokine-producing cells, possibly reflecting a higher activation state of their precursors. After expansion, antigen-specific Treg retain a classical T-reg phenotype (CD127- and FOXP3+) even upon exposure to pro-inflammatory stimuli, but contain a high proportion of $\mathrm{IFNg}^{+}$cells. Reduction of $\mathrm{IFNg}^{+}$cells in the presence of $\mathrm{RP}$ suggests a role for this drug in the expansion of antigen-specific T-reg for immunotherapy in AIH-2.

\section{P95 MOLECULAR MECHANISMS OF MONOCYTE REPROGRAMMING IN ACUTE LIVER FAILURE: IMPORTANCE OF HEPATICALLY DERIVED ANTI-INFLAMMATORY MEDIATORS}

doi:10.1136/gutjnl-2011-300857a.95

${ }^{1} \mathrm{C} G$ Antoniades, ${ }^{2} \mathrm{~V}$ Zingarelli, ${ }^{3} \mathrm{~L}$ Possamai, ${ }^{2} \mathrm{R}$ Mistry, ${ }^{2} \mathrm{R} \mathrm{D}$ Abeles, ${ }^{2} \mathrm{M}$ Bruce, ${ }^{1} \mathrm{M} J$ W McPhail, ${ }^{2} \mathrm{~N}$ Heaton, ${ }^{2} \mathrm{G}$ Auzinger, ${ }^{2} \mathrm{~W}$ Bernal, ${ }^{2} \mathrm{D}$ Vergani, ${ }^{3} \mathrm{M} \mathrm{R}$ Thursz, ${ }^{2} J$ A Wendon. 'Institute of Liver Studies, King's College Hospital, Denmark Hill, London \& Liver and Anti-viral Centre, St Mary's Hospital, Imperial College London; ${ }^{2}$ Institute of Liver Studies, King's College Hospital, Denmark Hill, London; ${ }^{3}$ Liver \& Anti-viral Centre, St Mary's Hospital, Imperial College London

Introduction Monocytes from patients with acetaminophen-induced acute liver failure (AALF) bear striking phenotypic and functional similarities with endotoxin tolerant (ET) monocytes and may account for the marked predisposition to sepsis and increased
NF-kBp65 expression:Controls vs ALF
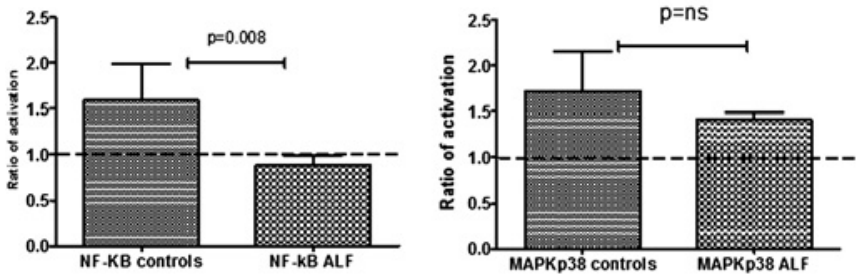

Abstract P95 Figure 1 Phosphoflow experiments investigating NFkBp65 and MAPKp38 expression following LPS challenge in study groups.

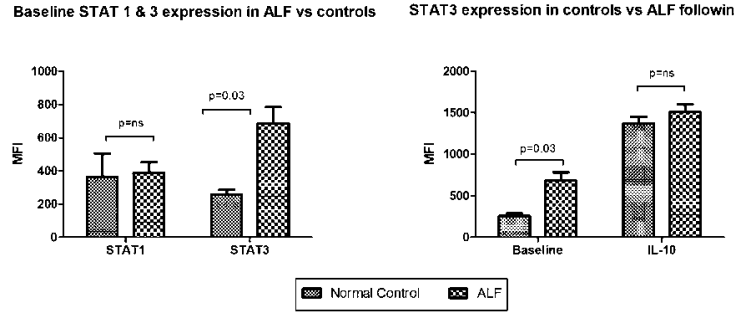

Abstract P95 Figure 2 Phosphoflow experiment investigating STAT-1 and STAT-3 expression in study groups at baseline and following exogenous IL-10 administration.

mortality in AALF. ET reprograms monocyte responses in response to lipolysaccharide (LPS) stimulation by reducing expression of proinflammatory (eg, TNF- $\alpha$, IL- 6 ) and augmenting the production of anti-inflammatory cytokines (eg, IL-10). At a molecular level, reductions in positive regulators (eg, NF-kBp65) of toll-like receptor (TLR)-4 dependent signalling pathway typify ET. Soluble antiinflammatory mediators, such as IL-10 and SLPI, exert negative regulation of TLR responses via STAT3 and NF-kBp65 dependent signalling pathways respectively.

Aim To delineate the molecular mechanisms and functional consequences of ET in AALF.

Method Following TLR-4 (LPS; $100 \mathrm{ng} / \mathrm{ml}), \operatorname{IFN}-\gamma(10 \mathrm{ng} / \mathrm{ml})$ and IL-10 $(50 \mathrm{ng} / \mathrm{ml})$ stimulation, phosphoflow technique was used to identify changes in regulators of TLR (NF-kBp65, MAPK p38), STAT-1 and STAT-3 signalling pathways in ex-vivo CD14+/CD33+ monocytes in eight AALF patients and 10 healthy volunteers (HC). Results expressed as MFI and ratio of activation (MFI following LPS/IFN- $\gamma /$ IL-10 stimulation divided by baseline MFI [RPMI]).

Serum TNF- $\alpha$, IL-10 and SLPI were measured by ELISA $(\mathrm{pg} / \mathrm{ml})$ in 34 AALF patients and 15 healthy volunteers (HC). Regional levels of TNF- $\alpha$, IL-10 and SLPI (portal vein [PV]), hepatic vein [HV]) were determined using in a further five AALF patients at time of liver transplantation (LT). Hepatic expression of TNF- $\alpha$, IL-10 and SLPI (all $\mathrm{pg} / \mathrm{ml}$ below) was determined using whole liver tissue homogenates from seven AALF explants and eight controls. Ex-vivo monocyte phagocytosis of FITC-labelled Escherichia coli was determined in five AALF and 10 healthy volunteers (HC) using FACS analysis.

Results In contrast to HC, TLR-4 stimulation markedly reduced NFkBp65 expression, while MAPKp38 signal transduction responses remained similar to that of $\mathrm{HC}$ (Abstract P95 figures 1-2). Baseline STAT-3 expression was significantly elevated in AALF patients compared to HC whereas no differences in STAT-1 expression was detected (Abstract P95 figure 2). Increase in STAT-3 expression following IL-10 stimulation was similar between AALF patients and HC.

AALF patients had significantly higher serum concentrations of TNF- $\alpha$ (21 vs $1.5 ; p<0.001)$, IL-10 (170 vs 40; $p<0.001)$ and SLPI (71 200 vs $43310 ; \mathrm{p}<0.001)$ compared to HC. A trans-hepatic (HV > PV) gradient was seen for IL-10 and SLPI but not for TNF- $\alpha$ in 4 out

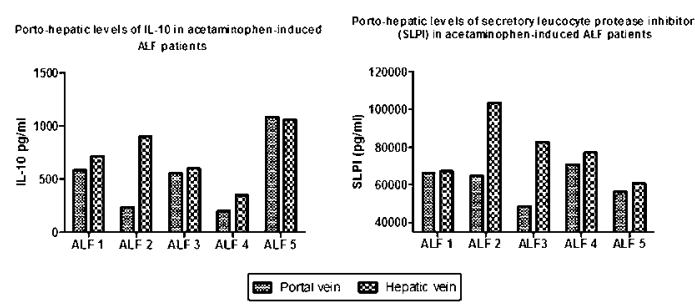

Abstract P95 Figure 3 Regional levels of SLPI and IL-10 in five patients with AALF. 
5 AALF patients sampled (Abstract P95 figure 3). Intra-hepatic levels of IL-10 ( 2 vs $0.6 ; p=0.03$ ) and SLPI (442 vs $116 ; p=0.004$ ) were higher in patients with AALF compared to controls, whereas no difference in TNF- $\alpha$ (24 vs 19; $\mathrm{p}=0.3$ ) concentration was detected. The percentage of monocytes phagocytosing $E$ coli was significantly reduced in ALF compared to HC (69 vs $92 \%$; $p=0.008$ ).

Conclusion In AALF, circulating monocytes show modulations in intracellular signalling pathways compatible with ET and display reduced phagocytic capabilities. Our data also indicate that hepatic production of anti-inflammatory mediators, IL-10 and SLPI, may play a pivotal role in induction of ET monocytes and thus increase the risk of infection in AALF.

\section{P96 SERUM PROTEIN N-GLYCOSYLATION AS A BIOMARKER OF PAEDIATRIC NAFLD}

doi:10.1136/gutjnl-2011-300857a.96

${ }^{1} \mathrm{E}$ Fitzpatrick, ${ }^{2} \mathrm{~B}$ Blomme, ${ }^{3} \mathrm{~A}$ Quaglia, ${ }^{4} \mathrm{R} \mathrm{D}$ Bruyne, ${ }^{2} \mathrm{H}$ V Vlierberghe, ${ }^{1} \mathrm{~A}$ Dhawan. ${ }^{1}$ Paediatric Liver, Gl and Nutrition Centre, King's College Hospital; ' ${ }^{2}$ Department of Gastroenterology and Hepatology, Ghent University Hospital, Belgium; 'Institute of Liver Studies, King's College Hospital; ${ }^{4}$ Department of Paediatric Gastroenterology, Hepatology and Nutrition, Ghent University Hospital, Belgium

Introduction Serum protein N-glycosylation has previously been shown to distinguish adult patients with simple steatosis from those with non-alcoholic steatohepatitis (NASH). The pattern of the disease in paediatric patients is distinct from adults.

Aim The aim of this study was to characterise the glycomic profile of children with varying degrees of NAFLD to identify potential biomarker profiles of disease.

Method Children with biopsy proven non-alcoholic fatty liver disease $(n=51)$ were recruited from a tertiary paediatric hepatology unit. Liver biopsy was scored according to NAFLD activity score. Blood was taken on day of biopsy for analysis. Serum protein $\mathrm{N}$-glycosylation patterns were assessed with DNA-sequencer assisted fluorophore-assisted capillary electrophoresis (DSA-FACE) and compared with histology.

Results Median age at biopsy was 13.3 years (range 4.5-17.4). 31 were male. Median BMI z-score was 1.81. 23 children scored as simple steatosis/borderline NASH and 28 as true NASH. 18 children had no/minimal fibrosis $(<$ F2) and 33 had significant fibrosis $(\geq$ F2). Similar to previous work in adult patients with NAFLD, peak 1 (NGA2F) was the most significantly raised N-glycan in paediatric NASH patients with peak 5 (NA2) demonstrating the greatest decrease. The logarithmically transformed ratio of peak 1 to peak 5 (Glycomarker) was -0.85 (SD 0.22) in simple steatosis/borderline NASH and -0.73 (SD 0.12) in NASH $(p=0.02)$. The biomarker correlated well with the amount of lobular inflammation with a consistent increase with ascending grade of inflammation. There was also a trend towards significance in differentiating patients with significant fibrosis $\geq \mathrm{F} 2 ;-0.74$ (SD 0.13) from patients with no/ minimal fibrosis $<F 2 ;-0.86$ (SD 0.24), $(p=0.06)$. Glyco-analysis of immunoglobulin $\mathrm{G}$ (IgG) confirmed the undergalactosylation status with a significant increase in peak 1 (NGA2F; $\mathrm{p}=0.024$ ) and a significant decrease of peak 6 (NA2F; $p=0.01$ ) on IgG. In multivariate analysis of the Glycomarker, GGT, AST and INR, only the Glycomarker displayed a significant result for distinguishing simple steatosis from NASH $(p=0.019)$.

Conclusion In conclusion, the findings in this study are novel in that they represent the first Glycomic analysis of paediatric NAFLD. They validate findings in adults in that a Glycomarker can serve reliably as a biomarker of severity of disease in NAFLD. The same N-glycosylation alterations are observed in paediatric NASH patients when compared to an adult population and therefore the same biomarker can be used. B cells play a dominant role in the $\mathrm{N}$-glycan alterations of NASH patients, both in an adult and paediatric population.

\section{P97 LYMPHOCYTE-HEPATOCYTE INTERACTIONS: HEPATITIS C VIRUS CHANGES THE RULES}

doi:10.1136/gutjnl-2011-300857a.97

${ }^{1} \mathrm{Z}$ Stamataki, ${ }^{1} \mathrm{O}$ S Qureshi, ${ }^{1} \mathrm{G} M$ Reynolds, ${ }^{2} \mathrm{~L}$ Hibbert, ${ }^{2} \mathrm{~J}$ Waters, ${ }^{2} \mathrm{G}$ R Foster ${ }^{3} \mathrm{~J} Z$ Rappoport, ${ }^{4} \mathrm{~S}$ G Hubscher, ${ }^{1} \mathrm{D}$ H Adams, ${ }^{1} \mathrm{~J}$ A McKeating. ${ }^{1} \mathrm{MRC}$ Centre for Immune Regulation, Institute of Biomedical Research, University of Birmingham, UK ${ }^{2}$ The Liver Unit, Queen Mary's University of London, UK; ${ }^{3}$ School of Biosciences, University of Birmingham, UK; ${ }^{4}$ Department of Pathology, University of Birmingham, Edgbaston, UK

Introduction Hepatitis C Virus (HCV) is a major cause of liver disease worldwide. Innate and adaptive cellular immune responses play a critical role in resolving acute HCV infection. However, the majority of infections are not cleared, resulting in a progressive chronic liver disease consistent with inadequate immune control. Evidence from human and animal models suggest that T cells play a critical role in controlling acute HCV infection, yet the mechanism (s) behind their failure to control chronic HCV replication are unknown. HCV replicates predominantly in the liver and virus specific immune cells need to target infected hepatocytes to control virus replication. HCV specific effector cells have been reported to home to the liver, however, little is known on their subsequent trafficking and fate within the organ.

Aim Our aim is to investigate the role of HCV infection on lymphocyte-hepatocyte interactions, migration and immune cell effector function.

Method We used in vitro and ex vivo models to study the effect of HCV infection on lymphocyte-hepatocyte interactions. Primary lymphocytes and hepatocytes were used in combination with hepatoma cell lines and replication competent HCV clones. Ex vivo lymphocyte migration assays were performed using biopsy material and tissue from explanted liver. Results were confirmed by in vivo observations using tissue sections from patients with end stage liver disease of viral and non-viral origin. Experimental techniques included immunohistochemistry, flow cytometry, fixed and live cell time-lapse confocal microscopy.

Results We demonstrate: (1) A role for hepatocyte ICAM-1 in mediating T-lymphocyte adhesion and migration; (2) T-lymphocytes migrate spontaneously through hepatocyte monolayers via cell-cell junctions; (3). HCV enhances T-cell transmigration and pro-inflammatory cytokine expression. Our data demonstrate the existence of novel interactions between T cells and hepatocytes that are modulated in HCV infection. The nature of the T cell-hepatocyte interactions may have an impact on T-cell effector function and the outcome of anti-viral immune responses.

Conclusion Interaction with HCV-infected hepatocytes alters T-cell trafficking and cytokine expression, providing a novel mechanism for HCV to persist in the liver.

\section{P98 THE EFFECTS OF $\mathbf{T}_{\text {H17 }}$ CYTOKINES ON LIVER PARENCHYMAL CELLS SHAPE THE MICROENVIRONMENT FOR LOCAL GENERATION OF $\mathrm{T}_{\mathrm{H17}} / \mathrm{T}_{\mathrm{C17}}$ IN INFLAMMATORY LIVER DISEASE}

doi:10.1136/gutjnl-2011-300857a.98

${ }^{1} \mathrm{E}$ H Humphreys, ${ }^{1} \mathrm{G}$ M Muirhead, ${ }^{1} \mathrm{R}$ H Bhogal, ${ }^{2} \mathrm{~B}$ Eksteen, ${ }^{1} \mathrm{~S}$ C Afford, Ye H Oo, ${ }^{1} \mathrm{D}$ H Adams. ${ }^{1}$ Centre for Liver Research, and NIHR Biomedical Research Unit, Institute of Biomedical Research, University of Birmingham, UK; ${ }^{2}$ Snyder Institute of Infection, Immunity and Inflammation, Health Research and Innovation Centre, Calgary, Canada

Introduction IL-17 secreting T cells (Th17 $\mathrm{h}_{\mathrm{h} 17}$ and $\left.\mathrm{Tc} 17_{\mathrm{c} 17}\right)$ are subsets of $\mathrm{T}$ lymphocytes that have been implicated in autoimmunity, inflammatory disease and provide a link between the innate and adaptive immune responses. High numbers of IL-17-producing T cells are found in close proximity to bile ducts in several liver diseases 